\title{
ARTICLE How well can morphology assess cell death modality? A proteomics study
}

\author{
Alexey L Chernobrovkin and Roman A Zubarev
}

While the focus of attempts to classify cell death programs has finally shifted in 2010 s from microscopy-based morphological characteristics to biochemical assays, more recent discoveries have put the underlying assumptions of many such assays under severe stress, mostly because of the limited specificity of the assays. On the other hand, proteomics can quantitatively measure the abundances of thousands of proteins in a single experiment. Thus proteomics could develop a modern alternative to both semiquantitative morphology assessment as well as single-molecule biochemical assays. Here we tested this hypothesis by analyzing the proteomes of cells dying after been treated with various chemical agents. The most striking finding is that, for a multivariate model based on the proteome changes in three cells lines, the regulation patterns of the 200-500 most abundant proteins typically attributed to household type more accurately reflect that of the proteins directly interacting with the drug than any other protein subset grouped by common function or biological process, including cell death. This is in broad agreement with the 'rigid cell death mechanics' model where drug action mechanism and morphological changes caused by it are bijectively linked. This finding, if confirmed, will open way for a broad use of proteomics in death modality assessment.

Cell Death Discovery (2016) 2, 16068; doi:10.1038/cddiscovery.2016.68; published online 3 October 2016

\section{INTRODUCTION}

Since the first descriptions of programmed cell death mechanisms in $1960 \mathrm{~s}^{1-7}$ and until 2000s, most attempts to classify cell death programs were based on morphological characteristics. In 1973, Schweichel and Merker proposed a classification of several cell death modalities, including 'type I cell death' associated with heterophagy, 'type II cell death' associated with autophagy and 'type III cell death' not associated with any digestion. ${ }^{8}$ According to modern terminology, these types correspond to apoptosis, autophagy and necrosis, respectively. ${ }^{9-11}$ The Nomenclature Committee on Cell Death (NCCD) has formulated in 2005 and 2009 recommendations for the definition of cell death morphotypes. ${ }^{12,13}$ At the same time, numerous attempts have been ongoing to classify cell death mechanisms according to biochemical assays rather than morphological characteristics. ${ }^{9,13,14}$ In 2012, NCCD has expressed the belief that the time has become appropriate for a novel systematic classification of cell death based on measurable biochemical features. ${ }^{15}$

This shift from morphology to biochemistry was intended to signify the relentlessly increasing knowledge of the biochemical features of distinct cell death subroutines. For a long time it has been assumed that morphologically similar states represent the activation of identical or at least similar lethal signaling cascades. ${ }^{15}$ The underlying assumption was the presence of a 'tight' link between the biochemical cascades and morphological changes. But later it has become clear that apparently similar cell death morphological patterns, as assessed by microscopy, can hide a great deal of biochemical heterogeneity. The NCCD has stated that the presence of specific morphological features is not sufficient to establish a causal link between a given process and cellular demise. ${ }^{15,16}$ This statement effectively postulates a 'loose' link between the biochemistry and morphology in cellular death.
Figure 1 illustrates the difference between the tight and loose link models. The tight model assumes a bijective link (one-to-one correspondence) between the cause and the effect, that is, between the drug applied and morphological changes in dying cell. Knowing the cell mechanics, this model permits one, at least in principle, to 'reverse-engineer' the death pathway based on the observed morphology. This, in turn, would allow one to identify the upstream area in the protein network that has triggered the corresponding biochemical processes, thus locating the drug target. The loose model makes such a possibility much less probable.

The question whether the cell death mechanics is loose or tight is significant, as the tight mechanics may greatly simplify drug target discovery, for example, by a combination of dynamics proteomics and pathway analysis. ${ }^{17}$ So far, direct comparison of these models has remained challenging, not least because it implies quantitative comparison between the morphology and biochemistry. While biochemistry can be quantitatively assessed by assays employing standards (although single-reaction biochemical readouts are deemed by NCCD to be poor indicators of a precise death modality), ${ }^{15,16}$ morphology assessment has been mostly qualitative and notoriously prone to operator-dependent (mis)interpretations.

A significant challenge for the model comparison is the everexpanding list of recognized distinct regulated cell death modes. While the 2005 NCCD report listed four main cell death types, 2012 NCCD classification included 13 entries: anoikis, autophagic cell death, caspase-dependent and caspase-independent intrinsic apoptosis, cornification, entosis, extrinsic apoptosis by death receptors and dependence receptors, mitotic catastrophe, necroptosis, netosis, parthanatos and pyroptosis. ${ }^{15}$ These modalities are not mutually exclusive: it was said that a specific cell death-related pathway may progress simultaneously with another cell death

Division of Physiological Chemistry I, Department of Medical Biochemistry and Biophysics, Karolinska Institutet, Scheelesväg 2, SE-17 177 Stockholm, Sweden. 
a

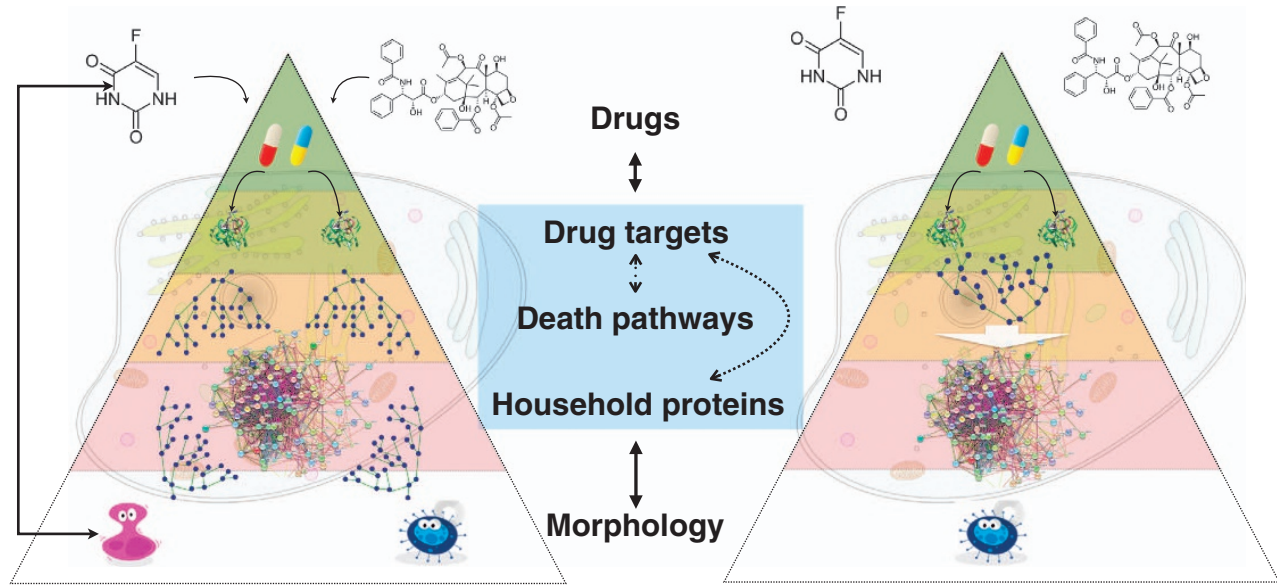

Figure 1. Tight or loose models of cell death regulation. Signal processing analogy can be used - stimulus as an input signal, cellular mechanisms as a black box, top proteome changes as an output signal. Left (a): In the tight model, different stimuli cause significantly different states of the dying cell, and such a state can be traced back to the actual cause of death. Right (b): In the alternative loose model, different lethal stimuli result in a common, or very similar, state of the dying cell, and based on that state, it is hard to decipher death's primary cause.

mode. $^{15}$ In a 2015 report, NCCD chose not to expand the evergrowing list of cell death modalities and the corresponding recommended assays to characterize them, noting that 'the best biochemical marker of cell death is death itself'. ${ }^{15}$

Such an abrupt conclusion may simply reflect the fact that the plurality of the discovered cell death modalities exceeded the specificity of the methods used for their assessments. A possible solution to this problem could be to increase the analytical specificity of the cell-death probing assay. Here we test whether proteomics can be such an enhanced assay. Indeed, modern proteomics can easily measure the relative abundance of $3000-5000$ proteins simultaneously ${ }^{18-21}$ or even of $\geq 10000$ proteins when larger sample volume and more instrumental time are available. $22-25$ The first few thousands of most abundant proteins constitute more than $90 \%$ of proteome mass, thus defining cellular structure and core functions, while the targets of common anticancer drugs are frequently found among the proteins in the low and middle abundance range. ${ }^{25,26}$ Therefore, a conventional 'top proteomics' analysis that takes $1-2 \mathrm{~h}$ of instrument time and probes $\leq 5000$ most abundant proteins encompasses both morphology-related proteins as well as druginteracting molecules. ${ }^{21,27}$

To evaluate the potential of top proteomics in differentiating between similar death modes, and possibly between the loose and tight cell death regulation models, we analyzed the proteomes of three human cancer cell lines after they were treated with five anticancer drugs - 5-fluorouracil (5-FU), methotrexate (MTX), tomudex (TDX), paclitaxel (PCTL) and doxorubicin (DOXO). ${ }^{28}$ These five drugs are widely used worldwide in anticancer therapy and their mechanisms have been studied extensively in last decades. For instance, there are 123613 papers indexed in Web Of Science (WoS) for 5-FU, 138613 for MTX, 87262 for PCTL, 144278 for DOXO and 915 for TDX. For comparison, for paracetamol and ibuprofen there are 29619 and 38692 items indexed in WoS, respectively. The panel of drugs encompasses such diverse (from the mechanism's standpoint) molecules as DNA and/or RNA synthesis inhibitors (5-FU and TDX), ${ }^{29-32}$ antifolate agent (MTX), ${ }^{33}$ tubulin-active antimitotic agent $(P C T L), 34,35$ and free radical formation and DNA damage agent (DOXO). ${ }^{36}$ Here we assess by means of proteomics whether or not the similarity in the formally assigned mechanism of action leads to the similarity in the death/survival pathways.

The protein abundance changes in dying cells were analyzed to reveal the proteins intimately involved in drug action, including the probable drug targets (set S). The 'causal' set S represents the upstream part of the cellular death mechanics, affection of which ultimately leads to cellular demise. Using the GO terms for various biochemical processes, proteins were also grouped into sets $B_{i}$, representing the proteins mediating the death cascades ( $i$ represented different $\mathrm{GO}$ terms). In parallel, the sets of $N$ most abundant proteins (sets $A_{N}$ ) were analyzed. These proteins, which are downstream of all death-related cascades, were taken to reflect the cellular morphological changes in the process of dying.

The two central questions of this study were: (i) which of the 'biochemical' sets $B_{i}$ reflects best the changes in the 'causal' set $S$ and (ii) how well is the 'morphological' set A reflects the behavior of set $S$ ? The hope was that, by answering the first question, we could evaluate the potential of proteomics for cell death modality discrimination. The second question would allow us to distinguish between the loose and tight models of cell death regulation, and simultaneously to verify the ability of 'quantitative morphology' (represented by the top proteome) to reflect the cell death mechanisms. In particular, the loose model predicts that at least some of the drugs would lead to practically indistinguishable morphological states of the cell, while the tight model suggests that all final cellular states will be well separated, and could potentially be traced back to the specific drug.

\section{RESULTS}

Verification of the approach

To verify that the S-set model for the three cell lines represents a good reference object, the protein 'loadings' of its OPLS-DA components were investigated (Figure 2). For the first (most significant) component that separates DOXO and PCTL on the one side from 5-FU, MTX and TDX on the other site (Figure 2a), the most typical DOXO representative was found to be ATP synthase ATP5A1, while the 5-FU champion was the RNA-binding motif protein RBM28. Furthermore, among the DOXO/PCTL-specific proteins, there is also a group of tubulins (PCTL targets), while TYMS (5-FU target) is found among the eight most 5-FU/TDX/MTXspecific proteins. The second OPLS-DA component separates the 5-FU and TDX/MTX treatments. Here, upregulation of eukaryotic translation initiation factor EIF4B and eukaryotic translation elongation factor EEF1B2, as well as downregulation of ribosomal protein RPL23A, were found to be most specific for 5-FU, while upregulation of DHFR (primary target for MTX and secondary target for TDX), TK1, CDK1 and PRIM1 were specific for TDX/MTX. 
a

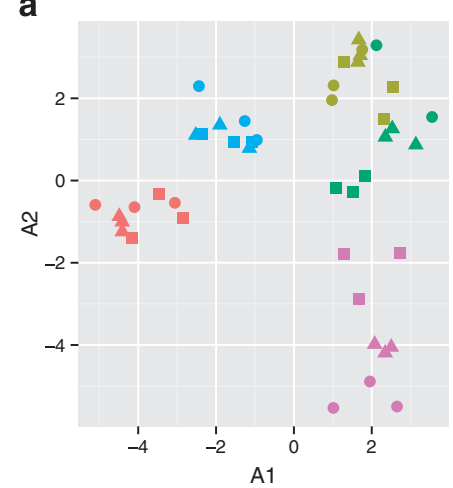

b

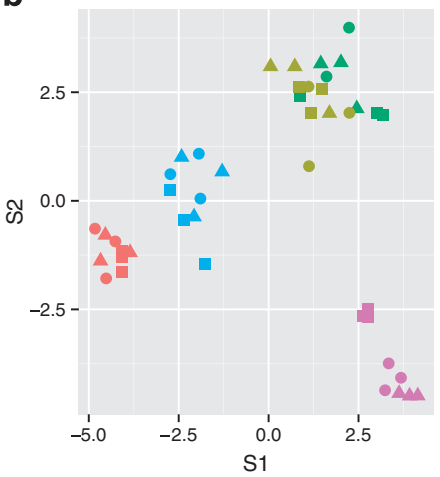

Cell $\bullet$ HCT116 $\boldsymbol{\Delta}$ H1299 $\square$ A375
Drug $\bullet$ DOXO $\bullet$ TDX $\bullet$ MTX $\bullet$ PCTL $\bullet$ 5-FU
C

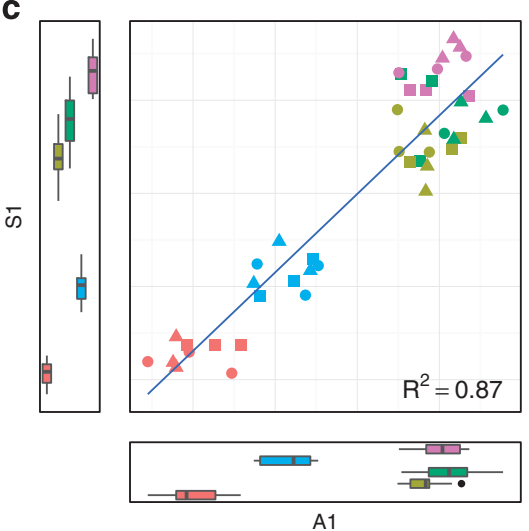

e

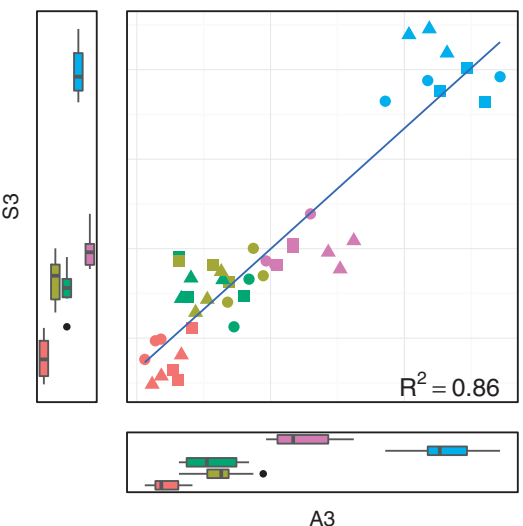

d

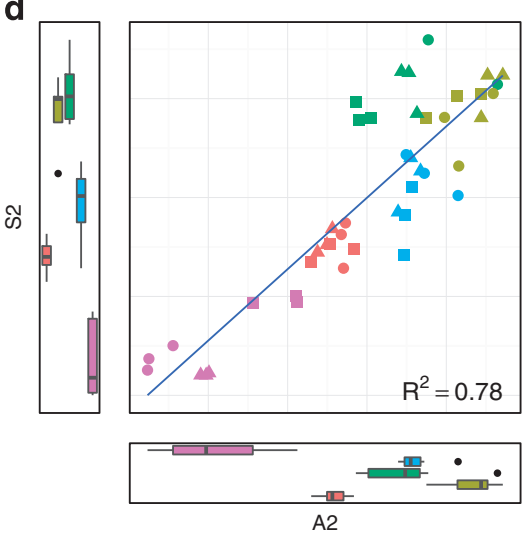

f

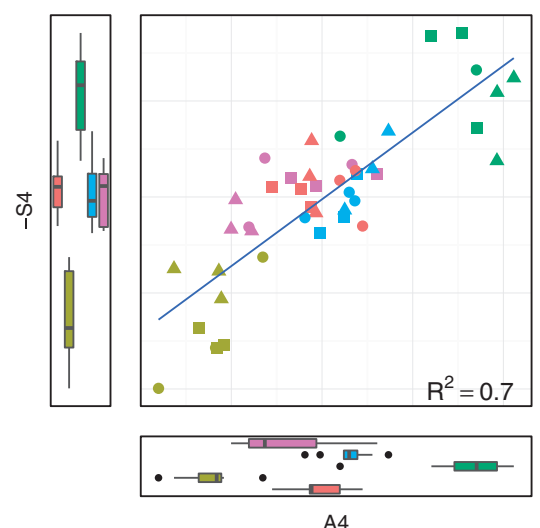

Figure 2. Comparison of two sets of proteins in terms of produced OPLS-DA models. (a) OPLS-DA model (first two dimensions are shown) separating different drug treatments for the $A_{100}$ set encompassing 100 most abundant proteins. (b) The same for the $S$-set encompassing 100 most specific proteins, including all primary and secondary drug targets. (c-f) Correlations between the first four coordinates of the OPLS-DA models of the $A_{100}$ set and $S$ sets.

Interestingly, TYMS was found in the middle group in this component, indicating that this protein is engaged in both 5FU- and TDX/MTX-induced pathways. The third component (Figure 2e) separates PCTL from DOXO; upregulated tubulins and downregulated BPTF were specific for PCTL, while the DOXOrelated proteins were CDK2 and SEC14L2. The fourth component (Figure 2f) differentiates TDX (the specific group of proteins includes CDK2, PRIM1, TK1 and TYMS) from MTX (SYNJ2, SEC14L2 and DHFR). These findings are in a broad agreement with existing knowledge on the targets and the mechanism of action of these drugs, ${ }^{27,29-32,34,35,37}$ which supports the validity of the S-setbased model.

As an example of the representativity estimate, the topologies of the OPLS-DA models for the sets $S$ and $A_{100}$ were compared (Figures $2 \mathrm{c}-\mathrm{f}$ ). The coordinates in all four orthogonal OPLS components correlate well with each other, with Pearson's correlation ranging from 0.81 to 0.93 . The representativity factor for this comparison was calculated to be 0.86 ( 0.7 after scaling).

\section{H1299 cell line}

Figure $3 a$ depicts the quality and representativity of various models based on the proteome changes in this cell line, while the model parameters are provided in Supplementary Table S2. All B-sets and A-sets for $N>2400$ fell into the shaded area representing random sets distribution ( $95 \%$ confidence). This fact indicates that even a randomly chosen proteomic set contains enough information to generate a good model separating the chosen treatments (mean $Q^{2}$ for random sets was 0.77 ). This, in turn, means that the information on the drug-induced effects is largely spread over the whole proteome. Yet the A-sets up to a moderate size $(N<2400)$ give better-quality models than any S-set.

Better performance of A-sets in terms of models' quality $\left(Q^{2}\right)$ can at least partially be explained by that fact that more abundant proteins are usually more accurately measured. In the Figure $3 \mathrm{~b}$ models' $Q^{2}$ are plotted against median CV of protein abundances in the data set. One can clearly see that the quality of the OPLS model decreases for the sets with higher median CVs. The good quality of A-sets in line with their relatively low representativity shows that morphological changes of cells under treatment are both drug-specific (good quality means that different treatments can be easily separated in the OPLS space) and cell-specific (upstream drug-specific perturbance of the proteome differs in terms of the OPLS topology from the downstream morphological changes). Thus, combining data on different cell lines could be away to separate drug-specific changes in morphology from cellline-specific proteome responses.

Three cell lines

Combining the data for all three cell lines minimized the cell-linespecific variations and thus highlighted the drug-specific protein abundance changes. In Figure $4 a$, only very few B-sets were found to behave better than the random sets (see also Supplementary Table S3). Among the significant GO terms are the regulation of cell death, regulation of apoptosis, RNA processing and translation, while the protein transport and protein localization sets 

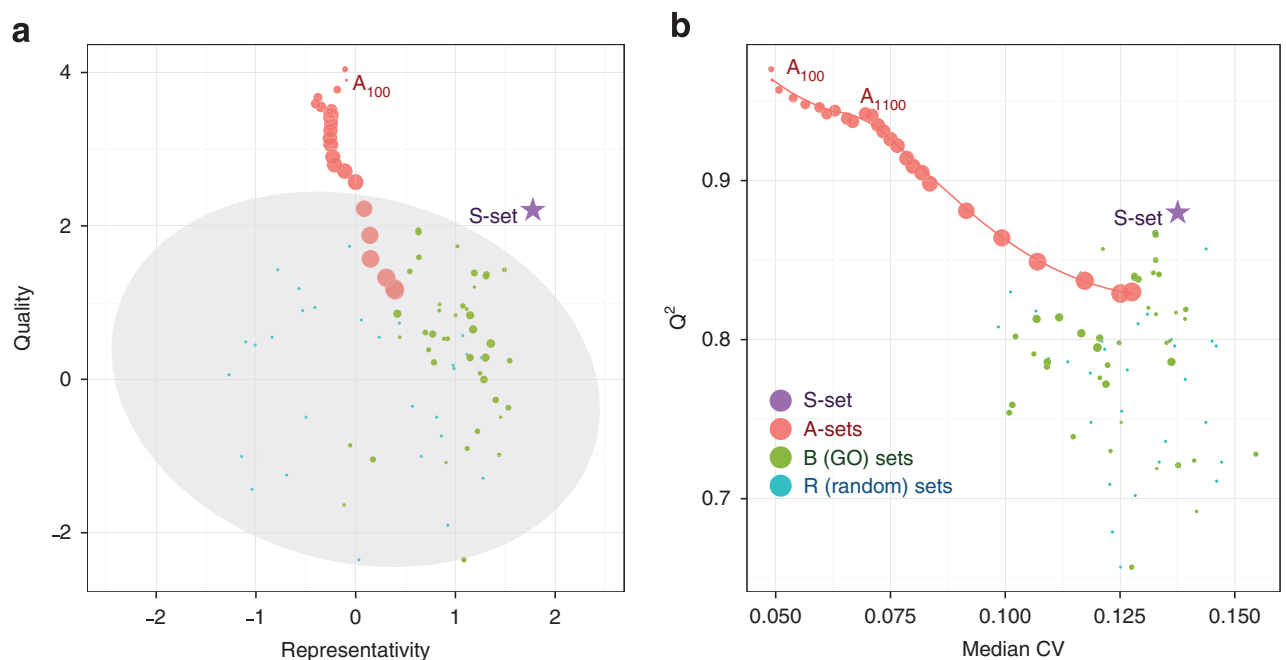

Figure 3. Quality and representativity of OPLS-DA models for different sets of proteins trained on proteomics data from H1299 cells treated with five drugs. (a) Normalized quality and representativity of different sets of proteins. The $95 \%$ confidence area for random sets is shown in gray. The circle sizes are proportional to the sizes of the protein sets. (b) Scatter plot of the OPLS-DA models' $Q^{2}$ values versus the median CV of protein abundances used in the model.
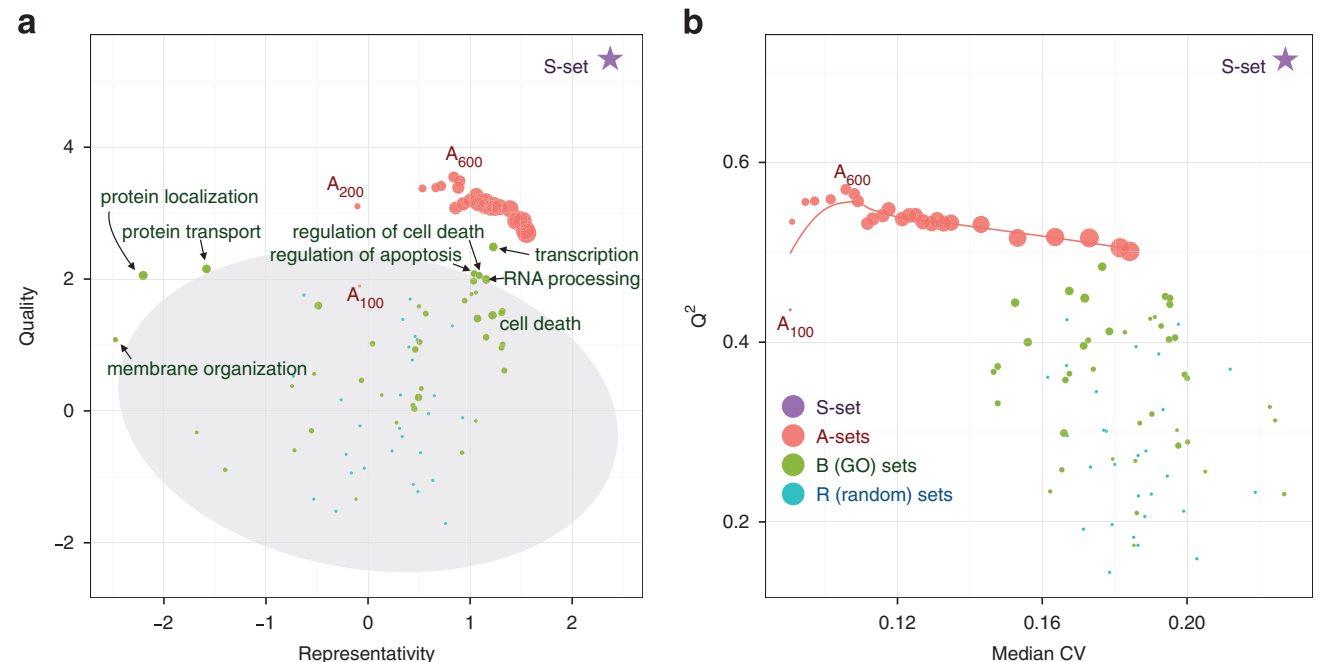

Figure 4. The same as in Figure 3 but for the combined data set on three cell lines (HCT116, A375 and H1299). (a) Normalized quality and representativity of different sets of proteins. The $95 \%$ confidence area for random sets is shown in gray. The circle sizes are proportional to the sizes of the protein sets. (b) Scatter plot of the OPLS-DA models' $Q^{2}$ values versus the median CV of protein abundances used in the model.

provided models with better quality but worse representativity. At the same time, all A-sets but one were found to be significant (better than random), while any A-set with $N \geq 200$ produced a better-quality model than any B-set.

As expected, the CVs for both single- and triple-cell data increased with $N$. However, unlike the single cell line data, the quality of OPLS models increased with $N$ for A-sets from 100 to 600 proteins and then decayed slowly (Figure 4b). There can be two reasons for this. First, combining data from three cell lines makes models more tolerant (to some extent) to experimental variations. Second, more proteins are needed for a combined data set to build a good model to separate drug-specific morphological changes from cell-line-specific changes.

\section{DISCUSSION}

To answer the first central question of this study, the set $B_{12}$ encompassing the GO term 'Transcription' reflected best the abundance changes of the set $S$, followed by $B_{7}$ and $B_{8}$ (deathrelated GO terms) as well as $B_{2}$ (RNA processing). While the presence of the death-related terms among the significant B-sets was anticipated, the prominence of the transcription and RNA processing B-sets was a bit surprising, though it could be explained by the fact that both transcription and RNA processing proteins are found in the highly abundant part of the cellular proteome. ${ }^{26}$ A larger panel of drugs, and perhaps of the cell lines, would allow one to identify which biochemically active protein sets most faithfully reflect the drug action mechanism.

The most striking finding in this study is the exceptionally good performance of the 'morphological' A-sets for combined data, which superseded the B-sets both in terms of the model quality as well as the representation of the drug action mechanism. Taken at the face value, this finding means that the regulation pattern of the abundant, household proteins faithfully reflects that of the proteins interacting with the drug, in a broad agreement with the rigid cell death model predictions. However, the final verdict on 
the rigid versus loose death mechanism can only be given when the number of tested drugs will greatly exceed that of the possible death pathways, that is, at least a few dozen.

If further research validates the rigid regulation model, top proteome analysis may become a valuable tool in drug development. Given that the quantitative analysis of the top proteome ( $\geq 1000$ proteins) can take as little as 10 min of instrumental time in a modern LC-MS/MS analysis, large-scale screening of drug action mechanisms may become possible.

\section{MATERIALS AND METHODS}

The data set for this study was taken from Chernobrovkin et al. ${ }^{28}$ In short, three cancer cell lines (melanoma A375, lung cancer H1299 and colon cancer HCT116) were treated in three biological replicates with five common anticancer agents, each one associated in literature with apoptosis: $5-\mathrm{FU}^{29,30,38,39}$ methotrexate (MTX), ${ }^{39}$ paclitaxel (PCTL), ${ }^{34,35}$ doxorubicin (DOXO) $)^{37,40}$ and tomudex (TDX). ${ }^{30}$ After $72 \mathrm{~h}$ treatment, most cells were dead (floating), with remaining cells dying but still attached. The attached dying cells were collected and lysed. Subsequent label-free proteomics analysis of cell lysates identified and reliably quantified 4168 proteins across all the treatments and cell lines. ${ }^{28}$ Most of the further analysis was done twice, first for the representative cell line (H1299) and then for the combined data on all three cell lines.

\section{Selection of set $\mathrm{S}$}

For each drug molecule, proteins were ranked according to the relation to the drug's mechanism of action and the corresponding $P$-values were calculated for each protein and drug as in ref. 28. For each individual protein, the $P$-values were multiplied, and the proteins were sorted in ascending order of the product. Top 100 proteins with the lowest products were selected as set S (Supplementary Table S1). The set S encompasses all known protein targets for the drugs used, for example, TYMS for 5-FU and TDX, DHFR for MTX, ß-tubulins for PCTL. ${ }^{28}$

\section{Selection of sets B}

Fourteen representative 'biochemical' sets (Table 1) were built using GO annotations (biological process) of groups encompassing 105-344 identified proteins. Protein identities in each B-set are given in Supplementary Table S1.

\section{Selection of sets $A$}

All quantified proteins were sorted according to the reference abundance (geometric mean of integrated ion current of all unique peptides for all cell types and treatments) ${ }^{41}$ in descending order. Subsets encompassing $N=100,200, \ldots, 4168$ proteins were created and subjected to further analysis.

\begin{tabular}{|lllc|}
\hline \multicolumn{2}{l}{ Table } & 1. & Selected B-sets \\
\hline Set & GO term & Description & Number of proteins \\
\hline$B_{1}$ & GO:0006281 & DNA repair & 105 \\
$B_{2}$ & GO:0006974 & Response to DNA damage & 138 \\
$B_{3}$ & GO:0000278 & Mitotic cell cycle & 165 \\
$B_{4}$ & GO:0016310 & Phosphorylation & 210 \\
$B_{5}$ & GO:0006928 & Cell motion & 122 \\
$B_{6}$ & GO:0007010 & Cytoskeleton organization & 148 \\
$B_{7}$ & GO:0007049 & Cell cycle & 288 \\
$B_{8}$ & GO:0008283 & Cell proliferation & 138 \\
$B_{9}$ & GO:0010941 & Regulation of cell death & 251 \\
$B_{10}$ & GO:0015031 & Protein transport & 344 \\
$B_{11}$ & GO:0006412 & Translation & 231 \\
$B_{12}$ & GO:0006350 & Transcription & 340 \\
$B_{13}$ & GO:0008219 & Cell death & 224 \\
$B_{14}$ & GO:0006915 & Apoptosis & 185 \\
\hline
\end{tabular}

Random sets

Sets of 100 proteins randomly chosen out of all quantified proteins were used to estimate the 'background level' of statistical significance of findings.

\section{GO annotation}

DAVID tool ${ }^{42}$ was used to extract gene ontology annotation for each quantified protein.

\section{Data sets comparison}

Multivariate approach was applied to reveal how different sets of proteins reflect cell behavior upon different treatments. The normalized and scaled protein abundances in each set were projected on latent structures using OPLS-DA ${ }^{43}$ method realized in SIMCA software (v. 13.5; Umetrics, San José, CA, USA). 'UV scaling' of variables was used leading to zero mean and unit variance for each protein across all samples. Classes were assigned to each sample according to drug treatment applied, namely 5-FU, DOXO, PCTL, MTX and TDX. Sevenfold crossvalidation was used to determine $Q^{2}$ of the model for each number of orthogonal components. ${ }^{44}$ The model based on the S-set was selected as a reference. All other models were compared with it in terms of the model quality (cross-validated $Q^{2}$ ) and 'representativity' of the drug action mechanism. The latter parameter was estimated as topology similarity with the set $\mathrm{S}$ model, and determined as follows. Projection coordinates (scores) for each component of the model were correlated with the corresponding coordinates of the S-set model. The mean value of the correlation coefficient between the two sets of components was chosen to be the 'representativity' estimate. Finally, quality and representativity values were normalized so as to provide zero means and unit variances for the models built upon random sets each comprising 100 randomly chosen proteins.

\section{ACKNOWLEDGEMENTS}

This work was supported by the Knut and Alice Wallenberg Foundation and Swedish Research Council.

\section{COMPETING INTERESTS}

The authors declare no conflict of interest.

\section{REFERENCES}

1 Fadeel B, Orrenius S. Apoptosis: a basic biological phenomenon with wideranging implications in human disease. J Intern Med 2005; 258: 479-517.

2 Lockshin RA, Williams CM. Programmed cell death-I. Cytology of degeneration in the intersegmental muscles of the Pernyi silkmoth. J Insect Physiol 1965; 11: 123-133.

3 Lockshin RA, Williams CM. Programmed cell death-II. Endocrine potentiation of the breakdown of the intersegmental muscles of silkmoths. J Insect Physiol 1964; 10: 643-649.

4 Lockshin RA, Williams CM. Programmed cell death-III. Neural control of the breakdown of the intersegmental muscles of silkmoths. J Insect Physiol 1965; 11: 601-610.

5 Lockshin RA, Williams CM. Programmed cell death. IV. The influence of drugs on the breakdown of the intersegmental muscles of silkmoths. J Insect Physiol 1965; 11: 803-809.

6 Lockshin RA, Williams CM. Programmed cell death. V. Cytolytic enzymes in relation to the breakdown of the intersegmental muscles of silkmoths. $J$ Insect Physiol 1965; 11: 831-844.

7 Lockshin RA, Beaulaton J. Programmed cell death. Life Sci 1974; 15: 1549-1565.

8 Schweichel JU, Merker HJ. The morphology of various types of cell death in prenatal tissues. Teratology 1973; 7: 253-266.

9 Lockshin RA, Zakeri Z. Apoptosis, autophagy, and more. Int J Biochem Cell Biol 2004; 36: 2405-2419.

10 Edinger AL, Thompson CB. Death by design: apoptosis, necrosis and autophagy. Curr Opin Cell Biol 2004; 16: 663-669.

11 Duprez L, Wirawan E, Vanden Berghe T, Vandenabeele P. Major cell death pathways at a glance. Microbes Infect 2009; 11: 1050-1062.

12 Kroemer G, El-Deiry WS, Golstein P, Peter ME, Vaux D, Vandenabeele P et al. Classification of cell death: recommendations of the Nomenclature Committee on Cell Death. Cell Death Differ 2005; 12(Suppl 2): 1463-1467. 
13 Galluzzi L, Aaronson Sa, Abrams J, Alnemri ES, Andrews DW, Baehrecke EH et al. Guidelines for the use and interpretation of assays for monitoring cell death in higher eukaryotes. Cell Death Differ 2009; 16: 1093-1107.

14 Van Cruchten S, Van den Broeck W. Morphological and biochemical aspects of apoptosis, oncosis and necrosis. Anat Histol Embryol 2002; 31: 214-223.

15 Galluzzi L, Vitale I, Abrams JM, Alnemri ES, Baehrecke EH, Blagosklonny MV et al. Molecular definitions of cell death subroutines: recommendations of the Nomenclature Committee on Cell Death 2012. Cell Death Differ 2012; 19: 107-120.

16 Galluzzi L, Bravo-San Pedro JM, Vitale I, Aaronson Sa, Abrams JM, Adam D et al. Essential versus accessory aspects of cell death: recommendations of the NCCD 2015. Cell Death Differ 2014; 22: 58-73.

17 Good DM, Zubarev Ra. Drug target identification from protein dynamics using quantitative pathway analysis. J Proteome Res 2011; 10: 2679-2683.

18 Cristobal A, Hennrich ML, Giansanti P, Goerdayal SS, Heck AJR, Mohammed S. In-house construction of a UHPLC system enabling the identification of over 4000 protein groups in a single analysis. Analyst 2012; 137: 3541.

19 Thakur SS, Geiger T, Chatterjee B, Bandilla P, Fröhlich F, Cox J et al. Deep and highly sensitive proteome coverage by LC-MS/MS without prefractionation. Mol Cell Proteomics 2011; 10: M110.003699.

20 Nagaraj N, Alexander Kulak N, Cox J, Neuhauser N, Mayr K, Hoerning O et al. System-wide perturbation analysis with nearly complete coverage of the yeast proteome by single-shot ultra HPLC runs on a bench top Orbitrap. Mol Cell Proteomics 2012; 11: M111.013722.

21 Pirmoradian M, Budamgunta H, Chingin K, Zhang B, Astorga-Wells J, Zubarev Ra. Rapid and deep human proteome analysis by single-dimension shotgun proteomics. Mol Cell Proteomics 2013; 12: 3330-3338.

22 Wilhelm M, Schlegl J, Hahne H, Gholami AM, Lieberenz M, Savitski MM et al. Mass-spectrometry-based draft of the human proteome. Nature 2014; 509: 582-587.

23 Geiger T, Wehner A, Schaab C, Cox J, Mann M. Comparative proteomic analysis of eleven common cell lines reveals ubiquitous but varying expression of most proteins. Mol Cell Proteomics 2012; 11: M111.014050.

24 Kim M-S, Pinto SM, Getnet D, Nirujogi RS, Manda SS, Chaerkady R et al. A draft map of the human proteome. Nature 2014; 509: 575-581.

25 Nagaraj N, Wisniewski JR, Geiger T, Cox J, Kircher M, Kelso J et al. Deep proteome and transcriptome mapping of a human cancer cell line. Mol Syst Biol 2011; 7: 548 .

26 Beck M, Schmidt A, Malmstroem J, Claassen M, Ori A, Szymborska A et al. The quantitative proteome of a human cell line. Mol Syst Biol 2011; 7: 1-8.

27 Marin-Vicente C, Lyutvinskiy Y, Romans Fuertes P, Zubarev RA, Visa N. The effects of 5-fluorouracil on the proteome of colon cancer cells. J Proteome Res 2013; 12: 1969-1979.

28 Chernobrovkin A, Marin-Vicente C, Visa N, Zubarev RA. Functional Identification of Target by Expression Proteomics (FITExP) reveals protein targets and highlights mechanisms of action of small molecule drugs. Sci Rep 2015; 5: 11176.

29 Longley DB, Harkin DP, Johnston PG. 5-Fluorouracil: mechanisms of action and clinical strategies. Nat Rev Cancer 2003; 3: 330-338.

30 Van Triest B, Pinedo HM, Giaccone G, Peters GJ. Downstream molecular determinants of response to 5 -fluorouracil and antifolate thymidylate synthase inhibitors. Ann Oncol 2000; 11: 385-391.
31 Burger K, Mühl B, Harasim T, Rohrmoser M, Malamoussi A, Orban M et al. Chemotherapeutic drugs inhibit ribosome biogenesis at various levels. $J$ Biol Chem 2010; 285: 12416-12425.

32 Peters GJ, Smitskamp-Wilms E, Smid K, Pinedo HM, Jansen G. Determinants of activity of the antifolate thymidylate synthase inhibitors Tomudex (ZD1694) and GW1843u89 against mono- and multilayered colon cancer cell lines under folaterestricted conditions. Cancer Res 1999; 59: 5529-5535.

33 Gorlick R, Bertino JR. Clinical pharmacology and resistance to dihydrofolate reductase inhibitors. In: Jackman AL (ed.). Antifolate Drugs in Cancer Therapy. Humana Press: Totowa, NJ, 1999, pp 37-57.

34 Horwitz SB. Mechanism of action of taxol. Trends Pharmacol Sci 1992; 13: 134-136.

35 Fan W. Possible mechanisms of paclitaxel-induced apoptosis. Biochem Pharmacol 1999; 57: 1215-1221.

36 Gewirtz DA. A critical evaluation of the mechanisms of action proposed for the antitumor effects of the anthracycline antibiotics adriamycin and daunorubicin. Biochem Pharmacol 1999; 57: 727-741.

37 Eom Y-W, Kim MA, Park SS, Goo MJ, Kwon HJ, Sohn S et al. Two distinct modes of cell death induced by doxorubicin: apoptosis and cell death through mitotic catastrophe accompanied by senescence-like phenotype. Oncogene 2005; 24: 4765-4777.

38 Nita ME, Nagawa H, Tominaga O, Tsuno N, Fujii S, Sasaki S et al. 5-Fluorouracil induces apoptosis in human colon cancer cell lines with modulation of $\mathrm{Bcl}-2$ family proteins. Br J Cancer 1998; 78: 986-992.

39 Herr I. Cellular stress response and apoptosis in cancer therapy. Blood 2001; 98 : 2603-2614.

40 Gamen $S$, Anel a, Pérez-Galán $P$, Lasierra $P$, Johnson D, Piñeiro a et al. Doxorubicin treatment activates a Z-VAD-sensitive caspase, which causes deltapsim loss, caspase-9 activity, and apoptosis in Jurkat cells. Exp Cell Res 2000; 258: 223-235.

41 Lyutvinskiy $Y$, Yang $H$, Rutishauser D, Zubarev R. In silico instrumental response correction improves precision of label-free proteomics and accuracy of proteomics-based predictive models. Mol Cell Proteomics 2013, 1-26.

42 Huang DW, Lempicki RA, Sherman BT. Systematic and integrative analysis of large gene lists using DAVID bioinformatics resources. Nat Protoc 2009; 4: 44-57.

43 Bylesjö M, Rantalainen M, Cloarec O, Nicholson JK, Holmes E, Trygg J. OPLS discriminant analysis: combining the strengths of PLS-DA and SIMCA classification. J Chemom 2006; 20: 341-351.

44 Westerhuis JA, Hoefsloot HCJ, Smit S, Vis DJ, Smilde AK, Velzen EJJ et al. Assessment of PLSDA cross validation. Metabolomics 2008; 4: 81-89.

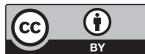

This work is licensed under a Creative Commons Attribution 4.0 International License. The images or other third party material in this article are included in the article's Creative Commons license, unless indicated otherwise in the credit line; if the material is not included under the Creative Commons license, users will need to obtain permission from the license holder to reproduce the material. To view a copy of this license, visit http://creativecommons.org/licenses/ by/4.0/

(c) The Author(s) 2016

Supplemental Information accompanies the paper on the Cell Death and Discovery website (http://www.nature.com/cddiscovery) 